\title{
Macrophage activation syndrome and COVID 19: Immune - Epigenetic programming in play
}

\author{
Roshan Kumar Roy ${ }^{1}$, Uttam Sharma ${ }^{2}$, Mishi Wasson ${ }^{1}$, Aklank Jain ${ }^{2}$, Md. Imtaiyaz \\ Hassan $^{3}$ and Hridayesh Prakash ${ }^{1}$ \\ ${ }^{1}$ Amity Institute of Virology and Immunology, Amity University NOIDA, U.P.India \\ ${ }^{2}$ Department of Zoology, Central University of Punjab, Bathinda, Punjab-151401, \\ India \\ ${ }^{3}$ Centre of Interdisciplinary Science, Jamia Millia Islamia, New Delhi-25, India
}

\section{*Address for correspondence}

\section{Dr. Hridayesh Prakash}

Amity Institute of Virology and Immunology,

Amity University, Sector-125, Noida, India

Phone: +91-120-4392961

Email: hprakash@amity.edu

Keywords: COVID-19, TLRs, Inflammasome, miRNA, LncRNAs, MAPK, Immune polarization 


\section{Summary}

Idiopathic association of the ongoing COVID-19 pandemic with many diseases appears to be multifactorial.SARS-CoV-2enters into host cells via ACE-II receptor and triggers the secretion of copious amount of IL-6;promote pulmonary fibrosis and Th2 / 17 programming of lungs, leading to severe lung pathology in COVID-19 patients. This virus interact and tweak all kind of cells like epithelium, macrophages, dendritic cells, and T cells and exploit them in a way that support its replication for progression of disease. Out of several pathophysiological manifestations, Macrophage activation syndrome is responsible for acute respiratory distress syndrome (ARDS) and subsequent death of COVID-19 patients. ${ }^{1}$ This is mainly accompanied by the increased infiltration of $\boldsymbol{F}_{C}{ }^{N_{1}}{ }^{+}$macrophages and other immune cells like neutrophils that contribute to Th2/Th17 programming of lung cells in COVID-19 patients. However, in view of durability and plasticity, macrophages are responsible for high-grade inflammatory response and mortality in patients. 


\section{State of Art}

Once derailed, hyper active alveolar macrophages secrete high levels of IFN-Y, IP10 (IP-10), IL-6, IL-17, TNF- $\alpha$ along with TGF- $\beta$ and IL-10 / 23 in severe cases of COVID-19. This prudently demonstrates MAS and the Th2/Th17 programming in the infectedlungs. $^{2}$ This is accompanied by the activation of inflammasome pathways which are crucial drivers of Th17 response. We believe that prolonged activation of CD14+ monocytes and phagocytosis of dead neutrophils by these cells contributes to NETosis in lung. This promotes Th2 bias in the lungs, decrease maturation of DC and monocytes, lymphocyte/neutrophils ratio and increases the risk of COVID-19 patients for death. In this context, in situ reprogramming of Th2/Th17 programmed macrophages towards M1phenotype macrophages is believed to afford protective immunity in COVID-19 cases ${ }^{1}$.

Professional immune cells like committed macrophage rely upon Toll like Receptors and associated pathways which are the guardian for Th1/2/17 effector responses in during any infection including SARS-CoV-2. ${ }^{3}$ Among various TLRs on macrophages, TLR-4, 5, 3, 7, and 9 actively sense spike proteins (N, S or $\mathrm{G}$ ) or mRNA of NSP-10, $\mathrm{S} 2$, and $\mathrm{E}$ proteins of SARS-CoV-2 and promote $\mathrm{M} 1$ polarization of macrophages ${ }^{4}$. Apart from ACE-2, the spike protein of SARS-CoV-2 uses TLR-2, 4 and 5 signaling pathways also via MyD88 and triggers Th1 effector response through NF-KB and ERK signaling cascade. ${ }^{5}$ Given this, tweaking TLR signaling cascades by using agonists can restore or promote Th1 response in derailed macrophages in COVID19 patients. Mimicking TLR-5 signaling can augment Th1 programming of macrophages and afford anti viral potential in these cells. Furthermore conjunction therapy with antivirals and TLR-7 agonists may benefit patients ${ }^{3}$ but whether this would reprogram macrophage also is open question and deserves attention. Combination of Tocilizumab and TLR-4 antagonists is believed to help in Th1 polarization of macrophage and in turn patients with severe disease displaying ARDS. Conjunction treatment of patients with Remdesivir with TLR-7 agonist is expected to overcome immune escape mechanism of viruses.

While promoting Th1 programming of infected macrophage is advantageous for host in controlling viral burden by the macrophages, balancing out cytokine storm, on other hand, in spite of being pre requisite yet a bottleneck for reducing fibrosis in patients. Several antagonists like M5049, hydroxychloroquine sulfate, FTY- 
720/ceramide-1 phosphate can competitively inhibit/alter the binding of spike proteins with TLRs and S-1P signaling respectively and believed to mitigate/arrest MAS and subsequently lung fibrosis. ${ }^{6}$ In this context, the administration of hydroxychloroquine in COVID-19 patients has shown to interfere with TLR signaling and mitigate inflammatory syndrome in patients. This is mainly due to interference of hydroxychloroquine in binding of DNA/RNA with endosomal TLRs with their ligands ${ }^{7}$.

Inflammatory programming of macrophage relies of several intracellular pathways like nk-kb / STAT / and p38MAPK which are associated with TLR. Of these p38MAPK pathway is one of the host factors implicated in lung and heart injury in COVID-19 patients. $^{8,9}$ P38MPAK is decisive for sterile inflammatory responses, desmoplastic reactions, and $\mathrm{T}$ cell exhaustion, epigenetic programming of host contributing to the severity of COVID-19 cases. Other than this, p38 MAPK controls macrophage's plasticity via promoting ER stress, unfolded protein responses, and glucose intolerance associated with energy imbalance in the infected host. SARSCoV-2 directly up regulates p38 activity directly for promoting its replication in epithelium and macrophages. ${ }^{10}$ The p38MAPK promotes Th2 bias, fibroblast activation significant for the SARS-CoV-2 induced aberrant inflammation in the lung. Therefore we strongly believe targeting p38MAPK could be of direct interest for not only tempering plasticity of infected macrophages, lowering pathogenic inflammatory pathways as well as T- cell exhaustion which is also seen in severely infected cases.

Most intriguingly p38MAPK are known to activate several intrinsic factors known as differentially expressed mi/lncRNAs for promoting Meta / sterile inflammation. These are tissue specific cues and influence both immunogenic / refractory pathways in host. IncRNA landscape is proposed as a prognostic factor responsible for the severity of COVID-19 cases. ${ }^{15}$ Among pool of miRNAs; miR-15b-5p, miR-15a-5p, miR-548c-5p, miR-548d-3p, miR-409-3p, miR-30b-5p and miR-505 have been validated as potent targets for controlling SARS-CoV-2 infection. ${ }^{16}$ These miRNAs regulate the expression of ACE-2 in various organs, including the kidney, heart, blood vessels, and lungs which are important for COVID-19 pathophysiology. ${ }^{17}$

Current evidence suggests that set of LncRNA like WAKMAR2, EGOT, EPB41L4AAS1, and ENSG00000271646, MALAT1, and NEAT1 contribute in skewing the immune response against SARS-CoV-2 infection. ${ }^{11,12}$ For instance NEAT1 
overexpression stabilizes the mature caspase- 1 to promote interleukin- $1 \beta$ production and modulate inflammasome activation ${ }^{13}$ which is associated with Th2/ 17 programming of immune cells. MALAT1 promotes Th1 effector responses and apoptosis in airway epithelial cells conditioned DCs and cardiac cells ${ }^{2,14}$ via miR-125b and p38MAPK / NF-KB pathways. ${ }^{3} T$ his loop is potentially involved in the maturation of monocyte / DC and their T cell programming ability which is essential for adaptive immunity of host.

\section{Perspective}

Thus, we believe that adjusting TLR / p38MAPK pathways in conjunction with epigenetic factors like IncRNA-miRNA would be a prudent approach not only for augmenting immunity of COVID-19 infected cases but also for enhancing their sensitivity for currently employed drugs. Taken together, such strategy would be helpful in curbing SARS-CoV-2 virus for managing COVID-19 disease.

\section{Conflict of Interest:}

Authors declare no conflict of interest. 


\section{References:}

1. Toor D, Jain A, Kalhan S, et al. Tempering Macrophage Plasticity for Controlling SARS-CoV-2 Infection for Managing COVID-19 Disease. Front Pharmacol 2020; 11: 570698.

2. Mehta P, McAuley DF, Brown M, et al. COVID-19: consider cytokine storm syndromes and immunosuppression. Lancet 2020; 395(10229): 1033-4.

3. Onofrio L, Caraglia M, Facchini G, Margherita V, Placido S, Buonerba C. Tolllike receptors and COVID-19: a two-faced story with an exciting ending. Future Sci OA 2020; 6(8): FSO605.

4. Choudhury A, Das NC, Patra R, Mukherjee S. In silico analyses on the comparative sensing of SARS-CoV-2 mRNA by the intracellular TLRs of humans. $J$ Med Virol 2021; 93(4): 2476-86.

5. Sariol A, Perlman S. SARS-CoV-2 takes its Toll. Nat Immunol 2021; 22(7): 801-2.

6. Prakash H, Upadhyay D, Bandapalli OR, Jain A, Kleuser B. Host sphingolipids: Perspective immune adjuvant for controlling SARS-CoV-2 infection for managing COVID-19 disease. Prostaglandins Other Lipid Mediat 2021; 152 : 106504.

7. In 't Veld AE, Jansen MAA, Ciere LCA, Moerland M. Erratum to "Hydroxychloroquine Effects on TLR Signalling: Underexposed but Unneglectable in COVID-19". J Immunol Res 2021; 2021: 9789246.

8. Grimes JM, Grimes KV. p38 MAPK inhibition: A promising therapeutic approach for COVID-19. J Mol Cell Cardiol 2020; 144: 63-5.

9. Hemmat N, Asadzadeh Z, Ahangar NK, et al. The roles of signaling pathways in SARS-CoV-2 infection; lessons learned from SARS-CoV and MERS-CoV. Arch Virol 2021; 166(3): 675-96.

10. Scott AJ, O'Dea KP, O'Callaghan D, et al. Reactive oxygen species and p38 mitogen-activated protein kinase mediate tumor necrosis factor alpha-converting enzyme (TACE/ADAM-17) activation in primary human monocytes. $J$ Biol Chem 2011; 286(41): 35466-76.

11. Vishnubalaji $R$, Shaath $H$, Alajez NM. Protein Coding and Long Noncoding RNA (IncRNA) Transcriptional Landscape in SARS-CoV-2 Infected Bronchial 
Epithelial Cells Highlight a Role for Interferon and Inflammatory Response. Genes (Basel) 2020; 11(7).

12. Mukherjee S, Banerjee B, Karasik D, Frenkel-Morgenstern M. mRNA-IncRNA Co-Expression Network Analysis Reveals the Role of IncRNAs in Immune Dysfunction during Severe SARS-CoV-2 Infection. Viruses 2021; 13(3).

13. Zhang $\mathrm{P}$, Cao L, Zhou $\mathrm{R}$, Yang $\mathrm{X}$, Wu $\mathrm{M}$. The IncRNA Neat1 promotes activation of inflammasomes in macrophages. Nat Commun 2019; 10(1): 1495.

14. Li Z, Zhang Q, Wu Y, et al. IncRNA Malat1 modulates the maturation process, cytokine secretion and apoptosis in airway epithelial cell-conditioned dendritic cells. Exp Ther Med 2018; 16(5): 3951-8.

15. Cheng J, Zhou X, Feng W, et al. Risk stratification by long non-coding RNAs profiling in COVID-19 patients. J Cell Mol Med 2021; 25(10): 4753-64.

16. Fulzele S, Sahay B, Yusufu I, et al. COVID-19 Virulence in Aged Patients Might Be Impacted by the Host Cellular MicroRNAs Abundance/Profile. Aging Dis 2020; 11(3): 509-22.

17. Lu D, Chatterjee S, Xiao K, et al. MicroRNAs targeting the SARS-CoV-2 entry receptor ACE2 in cardiomyocytes. J Mol Cell Cardiol 2020; 148: 46-9. 